\title{
Alle origini della tutela giuridica del consumatore: fondamenti romanistici della disciplina europea
}

\section{Premessa e linee espositive}

|| presente contributo si propone di individuare le radici romanistiche della tutela giuridica del consumatore nell'ambito della disciplina europea, mediante un'analisi comparativa di tipo diacronico tra la disciplina del diritto romano e quella del diritto di fonte europea.

Nello specifico, l'intento principale è quello di comparare taluni aspetti della disciplina della tutela del contraente debole nel contratto di compravendita, figura che, come è noto, attualmente coincide con quella del consumatore, concentrando l'attenzione su alcuni istituti presenti nel diritto romano, in cui emergono alcune situazioni di disparità contrattuale, che avevano richiamato prepotentemente l'attenzione degli Edili curuli in età repubblicana ${ }^{1}$. Nel corso dell'esposizione oltre a fare riferimento alla disciplina romanistica in tema di tutela del compratore a seguito dell'emanazione dell'Editto degli edili curuli De mancipiis emundis vendundis, si prenderà in considerazione la nozione di compratore/consumatore, nella sua accezione di contraente debole nell'ambito

\footnotetext{
* La Prof. Dr. Rosanna Ortu è professore associato di Istituzioni di diritto romano, Storia del diritto romano e di Fondamenti del diritto europeo presso il Dipartimento di Giurisprudenza dell’Università degli Studi di Sassari.E-mail: rortu@uniss.it rosannaortu@hotmail.com

${ }^{1}$ Cfr. G. Impallomeni, L'editto degli edili curuli, Padova 1955, 2. In generale, sul valore dell'intervento innovativo da parte degli edili curuli, rinvio anche alle interessanti osservazioni di F. Serrao, Impresa, mercato e diritto. Riflessioni minime, in mercati permanenti e mercati periodici nel mondo romano, Atti degli Incontri capresi di storia dell'economia antica, Capri 13-15 ottobre 1997, a cura di E. Lo Cascio, Bari, 2000, 33 ss., il quale ritiene che gli edili curuli con il loro editto contribuirono a formare un "diritto del mercato" che a partire dall'età repubblicana rappresenterà «un fenomeno anche esso, come quello pretorio, originalissimo».
} 
del contratto di compravendita, per poi concentrare l'attenzione sulla modalità di tutela configurata dal legislatore europeo (con riferimento specifico alla Direttiva 1999/44 CE), il quale ha dimostrato di intervenire sulla materia mediante una modalità simile a quella congeniata dagli Edili curuli in età repubblicana.

\section{La garanzia per vizi nell'editto degli edili curuli come esempio di tutela forte del contraente debole nel contratto di emptio venditio}

Come già anticipato nella premessa, l'indagine prenderà le mosse dalla differenza di tutela prevista nel diritto romano, specificamente nell'ambito del ius civile, a proposito del contratto di emptio venditio, tra la figura del venditor, sanzionato solo nell'ipotesi di reticenza o nel caso in cui sussistesse la responsabilità ex dicto vel promisso, e quella dell'emptor, al quale invece si concedeva la sola possibilità di intentare l'actio empti per il dolo del venditore.

Vale la pena sottolineare che nel diritto romano i rimedi forniti dal ius civile per sanzionare il venditore, a proposito dei vizi della cosa venduta, prevedevano unicamente il risarcimento del danno subìto dal compratore o la riduzione del prezzo. Le azioni contrattuali di ius civile pertanto, non erano mai dirette, almeno fino ad un certo periodo storico, alla risoluzione del contratto ${ }^{2}$ : un sistema di questo tipo non era quindi idoneo a tutelare il compratore, il quale si trovava a ricoprire senza alcun dubbio il ruolo di contraente debole nell'ambito del contratto di compravendita.

L'esigenza di tutelare i compratori dagli atti di frode dei mercanti di schiavi portò gli Edili curuli, magistrati che curavano e controllavano le vendite all'interno dei mercati, a creare una disciplina specifica in tema di compravendita e di garanzia per i vizi. Grazie a tale intervento, si introdusse una garanzia generale

${ }^{2}$ La dottrina non esprime parere univoco sulla possibilità che le azioni contrattuali potessero mirare alla risoluzione della compravendita e soprattutto sull'epoca a partire dalla quale si poteva applicare l'actio empti ad resolvendam emptionem. La dottrina tradizionale ritiene che siano rimaneggiati tutti i passi che prevedevano l'estensione dell'actio empti e, soprattutto, il frammento contenuto in D. 19.1.11.3 (Ulp. 32 ad ed.) in cui si afferma: Redhibitionem quoque contineri empti iudicio et Labeo et Sabinus putant et nos probamus. Tra gli altri, ritengono che questo passo sia interpolato V. Arangio-Ruiz, La compravendita in diritto romano ${ }^{2}$, II, Napoli 1956, 352 ss.; G. Impallomeni, L'editto degli edili curuli, cit., 244 ss. L'orientamento prevalente della dottrina più recente propende per considerare classico il contenuto del passo in questione e per ammettere, fin dall'epoca della giurisprudenza adrianea, l'esperibilità dell'actio empti per i vizi della cosa venduta in determinati casi: sia in concorso con le azioni edilizie, sia per estenderne la portata a ipotesi non tutelate dall'editto degli edili curuli. Sul punto vedi in part. M. Talamanca, voce Vendita (dir. romano), in ED, XLVI, Milano, 1993, 445. Di recente anche L. Manna, Actio redhibitoria e responsabilità per vizi della cosa venduta nell'editto de mancipiis emundis vendundis, Milano, 1994, 114 ss.; ma soprattutto L. Vacca, Ancora sull'estensione dell'ambito di applicazione dell'actio empti in età classica, in IURA, XLV, 1994, 248; N. Donadio, La tutela del compratore tra actiones aediliciae $e$ actio empti, Milano, 2004, $173 \mathrm{~s}$. 
del venditore per i vizi occulti della cosa venduta, inizialmente rappresentata dai soli schiavi e animali, dando vita così ad un sistema speciale che si preponeva all'impianto normativo della vendita del ius civile e che offriva particolare considerazione al compratore, con nuovi mezzi di tutela da esercitare contro il venditore, nei confronti del quale gravava una responsabilità di tipo oggettivo. Attraverso la concessione dell'actio redhibitoria ${ }^{3}$ venne perciò introdotto, per la prima volta, il principio della responsabilità generale ed oggettiva per i "vizi occulti" degli schiavi oggetto di vendita.

Tale attività normativa colmò gradualmente le lacune del ius civile. Il diritto edilizio, portio del ius honorarium ${ }^{4}$, iniziò a coesistere con la disciplina del ius

3 Tra i numerosi studi sull'actio redhibitoria rinvio soprattutto a: W.W. Buckland, The Roman law of slavery: the condition of the slave in private law from Augustus to Justinian, Cambridge 1908 (rist. 1969), pp. 59 ss.; R. Monier, La garantie contre les vices cachés dans la vente romaine, Paris, 1930, 59 ss.; A. Pezzana, D. 21, 1, 45. Contributi alla dottrina romana dell'actio redhibitoria, in RISG, serie III, 5, 1951, 275 ss.; F. Pringsheim, The decisive moment for Aedilician liability, cit., 545 ss.; V. Arangio-Ruiz, La compravendita in diritto romano, cit., 369 ss.; G. Impallomeni, L'editto degli edili curuli, cit., 137 ss.; A.M. Honoré, The history of the Aedilitian actions from Roman-dutch law, in Studi De Zulueta, Oxford 1959, 132 ss.; D. Pugsley, The Aedilician Edict, in Daube Noster, a cura di A. Watson, Edinburgh-London 1974, 253 ss.; A. Watson, Sellers' Liability for Defects: Aedilician Edict and Pretorian law, cit., 167 ss.; L. Manna, "Actio redhibitoria" e responsabilità per vizi, cit., 173 ss.; R. Zimmermann, The law of obligations. Roman Foundations of the Civilian Tradition, Oxford, 1996, 317 ss.; N. Donadio, Sull'"actio redhibitoria", in Index 25, 1997, pp. 649 ss.; L. Garofalo, "Redhibitoria actio duplicem habet condemnationem" (a proposito di Gai. ad ed. aed. cur. D. 21,1,45), in Atti del Convegno sulla Problematica contrattuale in diritto romano, Milano 11-12 maggio 1995. In onore di Aldo Dell'Oro, Milano, 1998, 57 ss.; ID., Perimento della cosa e azione redibitoria in un'analisi storico-compararatistica, in Europa e diritto privato 2, 1999, 843 ss.; ID., Studi sull'azione redibitoria, Padova, 2000, 1 ss.; E. Parlamento, Labeone e l'estensione della "redhibitio" all' "actio empti", in Rivista di Diritto Romano 3, 2003, pp. 1 ss. [http://www.led-online.it/rivistadirittoromano/]; R. Ortu, 'Aiunt Aediles'. Dichiarazioni del venditore e vizi della cosa venduta nell'editto de mancipiis emundis vendundis, Torino, 2008. Infine, a proposito di actiones aediliciae e actio empti, si veda lo studio di N. Donadio, La tutela del compratore, cit., 40 ss.

${ }^{4}$ Per l'appartenenza del diritto edilizio al ius honorarium rinvio a Gai 1.6: Ius autem edicendi habent magistratus populi Romani. Sed amplissimum ius est in edictis duorum praetorum, urbani et peregrini, quorum in provinciis iurisdictionem praesides earum habent; item in edictis aedilium curulium, quorum iurisdictionem in provinciis populi Romani quaestores habent: nam in provincias Caesaris omnino quaestores non mittuntur, et ob id hoc edictum in his provinciis non proponitur. Nelle fonti è possibile trovare anche un espresso riferimeno all'editto edilizio come parte integrante del ius honorarium, cfr. Inst. 1.2.7: Praetorum quoque edicta non modicam iuris optinent auctoritatem. haec etiam ius honorarium solemus appellare, quod qui honorem gerunt, id est magistratus, auctoritatem huic iuri dederunt. proponebant et aediles curules edictum de quibusdam casibus, quod edictum iuris honorarii portio est. La dottrina maggioritaria propende a ritenere che l'editto degli edili curuli fosse fonte di ius honorarium: al riguardo rinvio soprattutto a G. Impallomeni, L'editto degli edili curuli, cit., 1; ID., Edictum aedilium curulium, in Scritti di diritto romano e tradizione romanistica, Padova, 1996, (= in NNDI, VI, Torino, 1960, 372 ss.), 74 n. 2. Ma si veda anche A. Guarino, L'esaurimento del ius honorarium e la pretesa codificazione dell'editto, in Studi in memoria di E. Albertario, I, Milano, 1953, 625 ss; ID., L'editto edilizio e il diritto onorario, in Labeo, I, 1955, 295 ss.; ID., Ancora sull'editto edilizio, in Labeo, II, 1956, 352 ss.; il quale si oppone al pensiero espresso sul tema da E. Volterra, 
civile (che costituiva presupposto necessario per la responsabilità edilizia), e contribuì a formare un corpus di norme le quali, con nuove soluzioni giuridiche, disciplinarono un aspetto specifico del commercio degli schiavi che, in quel momento storico, rappresentava il fenomeno economico più rilevante.

L'abbondanza di opere e di studi sull'editto edilizio da parte dei giuristi romani fino al III sec. d.C. dimostra ulteriormente che, fin dall'inizio, l'intervento degli edili in materia di compravendite di servi, poi seguito da quello relativo alle vendite di animali, fu veramente innovativo ${ }^{5}$.

Per comprendere la portata innovativa del contenuto delle disposizioni edilizie, riporto qui di seguito il testo dell'editto de mancipiis emundis vendundis ${ }^{6}$ trascritto da Ulpiano in un lungo frammento, tratto dal suo commentario all'editto degli edili curuli:

\section{21.1.1.1 (Ulp. 1 ad ed. aed. cur.): Aiunt aediles: "Qui mancipia vendunt cer-} tiores faciant emptores, quid morbi vitiive cuique sit, quis fugitivus errove sit noxave solutus non sit: eademque omnia, cum ea mancipia venibunt, palam recte pronuntianto. Quodsi mancipium adversus ea venisset, sive adversus quod dictum promissumve fuerit cum veniret, fuisset, quod eius praestari oportere dicetur, emptori omnibusque ad quos ea res pertinet iudicium dabimus, ut id mancipium redhibeatur. Si quid autem post venditionem traditionemque deterius emptoris opera familiaeve procuratorisve eius factum erit, sive quid ex eo post venditionem natum adquisitum fuerit, et si quid aliud in venditione ei accesserit, sive quid ex ea re fructus pervenerit ad emptorem, ut ea omnia restituat. Item si quas accessiones ipse praestiterit, ut recipiat. Item si quod mancipium capitalem fraudem admiserit, mortis consciendae sibi causa quid fecerit, inve harenam depugnandi causa ad bestias intromissus fuerit, ea omnia in venditione pronuntianto: ex his enim

Intorno all'editto degli edili curuli, in Scritti in onore di U. Borsi, Padova, 1955, 3 ss.; ID., Ancora sull'editto edilizio, in IURA, VII, 1956, 141 ss. Posizione intermedia rispetto al pensiero dei due grandi Maestri è stata formulata da A. Pezzana, L'editto degli edili curuli (rec. critica a G. Impallomeni e E. Volterra), in IURA, VII, 1956, 249 ss. La dottrina più recente accetta pienamente la tesi del diritto edilizio come portio del ius honorarium. Vedi tra i più recenti studi L. Manna, Actio redhibitoria, cit., 4 s., in part. nt. 11.; ma soprattutto rinvio a R. Ortu, 'Aiunt Aediles', cit., 40 nt. 120.

${ }^{5}$ Cfr. V. Arangio-Ruiz, La compravendita, cit., 369, il quale, a proposito della concessione dell'azione redibitoria da parte degli edili, afferma: «In un sistema come quello romano, che ignora perfino la regola della risoluzione dei contratti per inadempimento, l'introduzione dell'azione in discorso rappresentava un'innovazione audace».

${ }^{6}$ L'editto degli edili curuli relativo alla vendita dei mancipia viene tradizionalmente denominato de mancipiis vendundis (cfr. O. Lenel, L'édit perpétuel, trad. fr. a cura di F. Peltier, Paris 1903, 303). Sulla scorta delle osservazioni di V. Arangio-Ruiz, La compravendita, cit., 362, in questo contesto preferisco utilizzare la dicitura "originaria", così come viene riprodotta nella tavoletta ercolanese TH 60. 


\section{causis iudicium dabimus. Hoc amplius si quis adversus ea sciens dolo malo vendidisse dicetur, iudicium dabimus"7.}

Il testo edittale, riprodotto e commentato da Ulpiano, appartiene sicuramente all'editto redatto in epoca adrianea dal giurista Salvio Giuliano.

Gli edili imposero ai venditori di schiavi l'obbligo di dichiarare ai compratori con voce chiara (palam recte pronuntianto ${ }^{8}$ ) i difetti fisici dello schiavo, se esso avesse la tendenza a fuggire o a vagabondare o se fosse a rischio di giudizio nossale. I venditori sarebbero stati considerati responsabili e passibili di actio redhibitoria qualora avessero contravvenuto a tali disposizioni o se avessero affermato che lo schiavo possedeva qualità in realtà assenti. Si stabiliva poi che era dovuto un risarcimento per il deterioramento dello schiavo provocato dal compratore, o dalla sua familia o dal suo procurator, e che la redibizione del mancipium comportava anche, qualora il servus non si fosse rivelato adatto al lavoro, la restituzione di tutti i frutti percepiti, a mezzo suo, dal compratore. Tutto ciò sarebbe avvenuto anche se l'emptor avesse scoperto che lo schiavo era stato condannato per un crimine capitale, o aveva tentato il suicidio o era

7 O. Lenel, Palingenesia iuris civilis, II, Lipsiae, 1889, 884, fr. 1758; ID., L'édit perpétuel, cit., 303, $\$ 293$. Su D. 21.1.1.1 (Ulp. 1 ad ed. aed. cur.) vedi C.F. Glück, Commentario alla Pandette, XXI, trad. it. a cura di S. Perozzi e P. Bonfante, Milano 1898, 13 ss.; P.F. Girard, Manuel Élèmantaire de Droit Romain, Paris, 1898, 551 ss.; W.W. Buckland, The Roman law of slavery, cit., 52 ss.; H. Vincent, Le droit des ediles. Étude historique et économique des prescriptions édiliciennes sur la vente et la garantie, Paris, 1922, 35 ss.; A. De Senarclens, La date de l'édit des édiles de mancipiis vendundis, in Tijd. 4, 1923, 388 ss.; R. Monier, La garantie contre les vices, cit., 32 ss.; F. Pringsheim, The decisive moment for Aedilician liability, cit., 546 ss.; G. Impallomeni, L'editto degli edili curuli, cit., 6 ss.; V. Arangio-Ruiz, La compravendita, cit., 362 ss.; B. Nicholas, Dicta promissave, in Studi De Zulueta, Oxford, 1959,91 ss.; A. Watson, The law of obligations, cit., 86 ss.; ID., The imperatives, cit., 73 ss.; ID., Sellers' Liability for Defects, cit., 168 s.; D. Pugsley, The Aedilician Edict, cit., 252 ss.; R. Zimmermann, The Law of Obligations, cit., 311 ss.; L. Manna, “Actio redhibitoria” e responsabilità per vizi, cit., 1 ss.; R. Cardilli, L'obbligazione di "praestare" e la responsabilità contrattuale in diritto romano (II sec. a.C. - II sec. d.C.), Milano, 1995, 134 s.; É. JAкав, Praedicere und cavere beim Marktkauf, München, 1997, 125 ss.; S. Randazzo, Leges mancipii, Leges mancipii, Milano, 1998, 126; R. Ortu, 'Aiunt Aediles', cit., 70 ss.

8 L'espressione 'palam recte' viene usata dagli edili anche nell'editto de iumentis vendundis riportato in D. 21.1 .38 pr. (Ulp. 2 ad ed. aed. cur.): Aediles aiunt: "qui iumenta vendunt, palam recte dicunto, quid in quoque eorum morbi vitiique sit ...". Il 'palam recte pronuntiare' e il 'palam recte dicere' nella realtà dei fatti si traducevano in una aperta denuncia dei vizi da parte del venditore, denuncia sottesa all'esigenza di chiarezza derivante dal principio della bona fides delle parti durante la contrattazione. Questa esigenza di chiarezza imposta dalla bona fides, per E. Betti, Istituzioni di diritto romano, II.I, Padova, 1960, 211: «da onere, la cui osservanza era deferita alla vigile iniziativa dei compratori, si convertiva in obbligo edittale destinato a garantire la sicurezza delle contrattazioni». Vedi anche A. Watson, The imperatives, cit., 73 ss.; S. Randazzo, Leges mancipii, cit., pp. 125 s., il quale sottolinea che l'espressione 'palam recte pronuntiando' dell'editto edilizio non può essere considerata tra quelle dichiarazioni che venivano a configurarsi come leges mancipii. Da ultima, sul tema, vedi R. Ortu, 'Aiunt Aediles', cit., 70 ss. 
stato condannato a combattere nell'arena. Infine, si stabiliva che sarebbe stata concessa l'azione edilizia in ogni caso in cui il venditore avesse agito con dolo.

Dalla lettura del frammento emergono i principi fondamentali della regolamentazione giuridica creata dagli edili per disciplinare la compravendita dei mancipia.

Attraverso l'editto viene introdotto, come già detto supra, il nuovo principio della responsabilità generale ed oggettiva per i vizi nelle vendite di schiavi. In seguito a ciò il venditore doveva dichiarare i vizi fisici, nonché alcuni particolari vizi morali e giuridici dello schiavo oggetto di vendita, e se non avesse ottemperato a tale obbligo sarebbe potuto incorrere, indipendentemente dalla buona o mala fede, nell'esperimento delle azioni edittali (il testo dell'editto riportato da Ulpiano fa menzione della sola azione redibitoria, che produceva effetti risolutori del contratto). L'editto prevedeva anche una responsabilità oggettiva ex dicto vel promisso, responsabilità già prevista dal ius civile, limitatamente ai casi in cui il venditore in buona fede avesse promesso determinate qualità o garantito l'assenza di vizi del mancipium con apposita stipulazione, il che comportava il solo risarcimento del danno subito dal compratore. Gli edili, però, prospettano anche in questo caso la possibilità di esperire l'azione redibitoria, che comportava la risoluzione del contratto, purché la fattispecie concreta presentasse i requisiti necessari per i quali fosse già possibile intentare l'azione di ius civile per il risarcimento del danno. Vi è quindi nell'editto un esplicito rinvio alla disciplina civilistica, che in questa particolare ipotesi costituiva il presupposto della responsabilità edilizia.

Gli edili poi introducono il principio della responsabilità per i danni causati dal compratore (o dai suoi sottoposti o dal suo procurator) e la restituzione dei frutti o accessori da lui guadagnati ad opera dello schiavo redibito.

Nell'editto è prevista anche una ulteriore responsabilità per il dolo del venditore: in base a tale principio era dolosamente responsabile chi, pur conoscendo il vizio, non lo avesse dichiarato al momento della vendita.

Gli edili concessero al compratore anche la possibilità di intentare l'actio aestimatoria contro il venditore, il quale avrebbe potuto essere condannato alla riduzione del prezzo di vendita nel caso in cui risultasse responsabile per l'omessa dichiarazione dei vizi occulti se la scoperta del vizio si fosse verificata da parte del compratore entro un anno dall'avvenuta compravendita.

L'Editto edilizio si configurava, pertanto, come una struttura composita il cui specifico campo di attuazione scaturiva dalla iurisdictio degli edili curuli, che si esplicava nei confronti delle vendite che si concludevano nei mercati. Le nuove disposizioni edilizie diedero vita ad una settorializzazione della materia, collocata comunque nella cornice normativa della vendita in generale. Scaturiva così una stratificazione in livelli della disciplina giuridica negoziale, in cui al

${ }^{9}$ Cfr. E. Betti, Istituzioni di diritto romano, cit., 211, il quale, a questo proposito, afferma che «la disciplina del ius civile è stata confermata e integrata dal diritto onorario». 
livello orizzontale si disponeva la disciplina di ius civile della compravendita, mentre a livello verticale ${ }^{10}$ si inseriva la regolamentazione speciale di ius honorarium introdotta dall'Editto degli edili curuli.

Il dettato edilizio, che, come appena ribadito, nel sistema giuridico romano trovò la sua origine e collocazione nell'ambito del ius honorarium, ha oltrepassato i confini temporali dell'epoca romana e influenzato le esperienze giuridiche successive: richiamato preminentemente sia dal diritto medievale ${ }^{11}$, sia dalle prime codificazioni dell'Europa continentale ${ }^{12}$.

${ }^{10}$ A tale proposito rinvio all'ipotesi ricostruttiva di F. Serrao, Impresa, Mercato, Diritto, cit., 33.

11 Nel medioevo la responsabilità per vizi della cosa, come sostenuto da H. Dilcher, Die Theorie der Leistungsstörungen bei Glossatoren, Kommentatoren und Kanonisten, Frankfurt, 1960, 284, venne «lentamente svincolata dai diversi fondamenti di responsabilità tipici del diritto antico», riconducendo la sua disciplina sotto un'unica fattispecie legale, che avesse come presupposto il vitium e in virtù di considerazioni di tipo equitativo. In questo particolare momento storico si assiste, nonostante il perdurare della esperibilità delle azioni edilizie, alla generalizzazione delle regole da parte dei glossatori, come nel caso della c.d. laesio enormis, che dava la possibilità sia al venditore, sia al compratore di pretendere la rescissione della compravendita quando il pretium era fortemente inadeguato all'oggetto. Per tutta la stagione del diritto comune, che si protrasse sino al declinare del secolo XVIII, i principi elaborati dalla giurisprudenza romana in materia di compravendita, specialmente con riferimento ai rimedi a favore del compratore delineati sull'intervento della magistratura edilizia, mantennero un'inesauribile vitalità, incontrando solo il limite dei preminenti iura propria, nel caso in cui, con apprezzabili differenze nell'intera Europa continentale, questi ultimi avessero disposto diversamente. Sul punto vedi anche G. Wesenberg, G. Wesener, Storia del diritto privato in Europa, Padova, 1999, 65; P. Stein, Medieval discussions of the buyer's actions for physical defects, in Studies in the Roman Law of Sale dedicated to the memory of F. De Zulueta, a cura di A. Daube, Oxford, 1959, 105; P. Sciuto, Sulla c.d. rescissione per lesione enorme, in Labeo, XLVI, 2000, 404 ss.; R. Westbrook, The Origin of Laesio Enormis, in RIDA, LV, 2008, 39 ss.

vi sono Autori come R. Lederle, Mortus redhibetur. Die Rückabwicklung nach Wandlung im römischen Recht (Schriften zur Rechtsgeschichte), Berlin, 1983, 34, che ritengono che non pochi usi locali avessero cancellato l'actio redhibitoria dai rimedi idonei ad assoggettare il venditore per i vizi della cosa venduta. Contra L. Garofalo, Studi sull'azione redibitoria, cit., 84 nt. 18, il quale afferma che tale posizione è rimasta isolata in dottrina e non adeguatamente dimostrata. Inoltre, vale la pena ricordare brevemente, l'esperienza giuridica tedesca dove l'incontro tra il diritto romano e i diritti locali germanici delimitò, sotto decisivo influsso di quest'ultimo, il campo di efficacia del rimedio redibitorio. Per quanto invece attiene alla tutela del compratore nelle legislazioni statutarie, si può affermare che questa era affidata all'azione redibitoria e all'azione estimatoria. Come sostenuto da R. Fubini, La teoria dei vizi redibitori nel diritto civile e commerciale italiano, Torino, 1896, «la legislazione statutaria [rimise] in onore la dottrina dell'editto edilizio» - pur, ovviamente, astraendo dal contesto socio-economico nel quale le azioni edilizie si sono sviluppate - accettando di buon ordine quei principi del diritto germanico «relativi alla concezione più severa ed umana nel medesimo tempo della responsabilità di chi addiviene a contrarre». L'atteggiamento di diffidenza del diritto romano nei confronti del venditore venne moderato dal diritto germanico, che richiedeva al venditore quel minimo di diligenza e prudenza nell'esaminare la merce prima della conclusione del contratto. Il diritto germanico, trattandosi di un diritto fondato in gran parte sulle consuetudini, si adattò efficacemente alle esigenze locali, soprattutto se si considera l'àmbito entro il quale le contrattazioni avvenivano, che coincideva prevalentemente con quello delle compravendite di animali

${ }^{12}$ In particolar modo si rinvia alle codificazioni di Germania, Austria, Francia e Italia. Per un'analisi esaustiva sul tema, si vedano R. Fubini, La teoria dei vizi redibitori, cit., 90 ss.; L. Garofalo, Le 


\section{Dal compratore al consumatore: La Direttiva 1999/44/CE}

Un possibile collegamento tra il diritto romano e le nuove esperienze europee contemporanee può essere riscontrato non solo in riferimento ai mezzi di tutela concessi al compratore/consumatore, ma anche nella considerazione del c.d. elemento della 'verticalità' ${ }^{13}$ della disciplina edilizia rispetto alla disciplina di carattere generale della compravendita di ius civile. Tale elemento della 'verticalità', che comunemente coincide con un provvedimento esterno, rivolto alla creazione di categorie dogmatiche, ha raffigurato attualmente la modalità di intervento utilizzata, nei vari ordinamenti giuridici europei nonché dal legislatore europeo, per fornire una regolamentazione speciale in materia di garanzie nella vendita, più attenta alle necessità e alla condizione giuridica del compratore/consumatore.

Dallo studio dei più recenti interventi del legislatore europeo, infatti, emerge che nell'ambito del processo di armonizzazione del diritto degli Stati membri, si è mostrato interesse a quelle particolari situazioni di squilibrio contrattuale riscontrabili nella compravendita, contratto in cui il venditore è pervenuto ad un elevato valore di professionalità che lo colloca in una condizione di forte vantaggio rispetto al compratore. Così come si verificava nell'antica Roma, il compratore odierno, dinnanzi a tale forza contrattuale del venditore, versa in una condizione di debolezza soprattutto in merito alla tipologia dei mezzi di

azioni edilizie, cit., 491 ss.; P.M. Vecchi, La garanzia nella vendita in diritto comune, in Verbraucherkauf in Europa: Altes Gewährleistungsrecht und die Umsetzung der Richtlinie 1999/44/EG, a cura di M.J. Schermaier, Monaco, 2003, 85 s. In merito alla codificazione francese rinvio inoltre a R.J. Pothier, Traité du contrat de vente, in OEuvres de Pothier, III, Paris, 1825, 128, il quale, con riferimento al $\$$. 203, afferma che : «Le vendeur, par la nature du contrat de vente, est tenu de garantir l'acheteur, que la chose vendue est exempte de certains vices qui sont de nature à rendre ou presque inutile, ou même quelquefois nuisible, l'usage pour lequel cette chose est dans le commerce»; R.T. Troplong, Le droit civil expliqué suivant l'ordre du Code. De la vente ou commentaires du Tit. VI du Liv. III du Code civil, Bruxelles, 1836, 221. Si veda anche V. Mannino, Questioni di diritto, Milano, 2007, 196. Per la codificazione italiana e per la tutela del compratore e i vizi redibitori, vedi C. Ghisalberti, $L a$ codificazione del diritto in Italia: 1865-1942, Roma-Bari, 1985, 38 ss.; R. Montessori, La garanzia del venditore pei vizi della cosa e la denuncia dei difetti della merce nella compravendita commerciale, Milano, 1910, 3 s.; E. Pacifici-Mazzoni, Trattato della vendita, in Il codice civile italiano commentato con la legge romana, le sentenze dei dottori e la giurisprudenza, a cura di E. Pacifici-Mazzoni, XII, Torino, 1929, 411 s. Tra i più recenti, vedi A. Luminoso, voce Vendita, in Digesto IV, Disc. privatistiche, Sez. civile, XIX, Torino, 1999, 278 s.; L. Savanna, La compravendita. Garanzia per vizi della cosa venduta e nuova tutela del consumatore, Torino, 2007, 7. In particolare sul tema dell'aliud pro alio si veda E. Gabrielli, La consegna di cosa diversa. L'aliud pro alio nei negozi giuridici: profili ricostruttivi, tendenze giurisprudenziali e tecniche interpretative, Napoli, 1987.

${ }_{13}$ Cfr. F. Serrao, Impresa, Mercato, Diritto. Riflessioni minime, cit., 33, il quale inoltre osserva che «la formazione, la natura e la funzione di un diritto del mercato» rappresentano l'effettivo ambito di intervento degli edili curuli. Da questa considerazione e dallo studio dell'elemento della 'verticalità' prende vita l'idea di un parallelo tra il diritto romano e le attuali esperienze giuridiche europee. 
tutela, dovendo avere a che fare il più delle volte con condizioni generali di contratto preordinate unilateralmente dall'altra parte contrattuale.

A questo punto si rende però necessario rivolgere l'attenzione alla specifica nozione di compratore/consumatore. Al riguardo, vale la pena sottolineare che l'attribuzione di un significato univoco al concetto di compratore/consumatore risulta essere abbastanza complicato, in quanto tale concetto recepisce caratteristiche proprie che derivano da differenti ambiti disciplinari, come quello economico, sociologico e giuridico ${ }^{14}$.

Nella Direttiva 1999/44/CE (avente per oggetto il ravvicinamento delle disposizioni legislative, regolamentari e amministrative degli Stati membri relative a taluni aspetti della vendita e delle garanzie concernenti i beni di consumo, al fine di garantire un livello minimo uniforme di tutela dei consumatori nel quadro del mercato interno) il legislatore europeo ha cercato di forgiare una definizione di consumatore ${ }^{15}$. Nell'art. 1 lett. a) della suddetta

${ }_{14}$ Cfr. M.P. Mantovani, La vendita dei beni di consumo, Napoli, 2009,41.

15 A proposito della Direttiva 1999/44/CE e della nozione di consumatore rinvio a C. Castronovo, S. Mazzamuto, Manuale di diritto privato europeo, Milano, 2007, 266; M.P. Mantovani, La vendita dei beni di consumo, cit., 34 . Diverse direttive comunitarie contengono una definizione del termine 'consumatore', seppur con alcune differenze nella redazione del testo. Il punto di partenza è rappresentato dalla definizione contenuta nell'art. 2 della Direttiva 1985/577/CEE del Consiglio, del 20 dicembre 1985, per la tutela dei consumatori in caso di contratti negoziati fuori dei locali commerciali (in G.U.C.E., n. L 372, del 31 dicembre 1985, pp. 31 ss.), ora abrogata dalla Direttiva 2011/83/UE, che definiva il consumatore 'la persona fisica che [...] agisce per un uso che può considerarsi estraneo alla propria attività professionale'. Identica definizione era contenuta nell'art. 1, §. 2 della Direttiva 1987/102/CEE del Consiglio, del 22 dicembre 1986, relativa al ravvicinamento delle disposizioni legislative, regolamentari e amministrative degli Stati Membri in materia di credito al consumo (in G.U.C.E., n. L 42, del 12 febbraio, 1987, pp. 48 ss.), ora abrogata e sostituita dalla Direttiva 2008/48/CE del Parlamento europeo e del Consiglio, del 23 aprile 2008, relativa ai contratti di credito ai consumatori e che abroga la Direttiva 1987/102/CEE (in G.U.U.E., n. L 133/66, del 22 maggio 2008), la che fornisce all'art. 3, let. a) la seguente definizione di consumatore: 'una persona fisica che $[. .$.$] agisce per scopi estranei alla sua attività commerciale o professionale'. Nella$ Direttiva 1993/13/CEE del Consiglio, del 5 aprile 1993, concernente le clausole abusive nei contratti stipulati con i consumatori (in G.U.C.E., n. L 95, del 21 aprile 1993, 29 ss.), il consumatore è definito all'art. 2, let. b) come 'qualsiasi persona fisica che [...] agisce per fini che non rientrano nel quadro della sua attività professionale’. Una definizione non difforme era contenuta nell'art. 2 , \$. 2 della Direttiva 1997/7/CE del Parlamento europeo e del Consiglio, del 20 maggio 1997 (in G.U.C.E., n. L 144, del 4 giugno 1997), ora abrogata dalla Direttiva 2011/83/UE, e nell'art. 2, let. d) della Direttiva 2002/65/CE del Parlamento europeo e del Consiglio, del 23 settembre 2002, concernente la commercializzazione a distanza di servizi finanziari ai consumatori e che modifica la Direttiva 1990/619/ CEE del Consiglio e le Direttive 97/7/CE e 98/27/CE (in G.U.C.E., n. L 271/16, del 9 ottobre 2002). La definizione di 'consumatore' più aggiornata è contenuta nell'art. 2, \$. 1 della Direttiva 2011/83/ UE del Parlamento europeo e del Consiglio, del 25 ottobre 2011, sui diritti dei consumatori, recante modifica della Direttiva 1993/13/CEE del Consiglio e della Direttiva 1999/44/CE del Parlamento europeo e del Consiglio e che abroga la Direttiva 1985/577/CEE del Consiglio e la Direttiva 1997/7/ CE del Parlamento europeo e del Consiglio (in G.U.U.E., n. L 304/64, del 22 novembre 2011), alla stregua del quale è consumatore 'qualsiasi persona fisica che [...] agisca per fini che non rientrano 
direttiva ${ }^{16}$, la figura del consumatore coincide con "qualsiasi persona fisica che, nei contratti soggetti alla presente direttiva, agisce per fini che non rientrano nell'ambito della sua attività commerciale o professionale".

La definizione di consumatore, pertanto, stando alla previsione letterale dell'art. 1, let. a) della Direttiva 1999/44/CE sopra citato, è stata delineata in maniera restrittiva, con connotazioni in negativo: il legislatore europeo delimita, infatti, il concetto alle sole persone fisiche; attribuendo in negativo tale qualificazione solo a chi agisce per finalità che stanno al di fuori della propria attività commerciale o professionale ${ }^{17}$.

nel quadro della sua attività commerciale, industriale, artigianale o professionale'. Quest'ultima Direttiva propende all'estensione di tale figura, così come risulterebbe alla lettera del Considerando n. 17: «La definizione di consumatore dovrebbe includere le persone fisiche che agiscono al di fuori della loro attività commerciale, industriale, artigianale o professionale. Tuttavia, nel caso di contratti con duplice scopo, qualora il contratto sia concluso per fini che parzialmente rientrano nel quadro delle attività commerciali della persona e parzialmente ne restano al di fuori e lo scopo commerciale sia talmente limitato da non risultare predominante nel contesto generale del contratto, la persona in questione dovrebbe altresì essere considerata un consumatore».

${ }_{16}$ Si veda il testo art. 1 della Direttiva 1999/44/CE, in cui si legge: 1. La presente direttiva ha per oggetto il ravvicinamento delle disposizioni legislative, regolamentari e amministrative degli Stati membri relative a taluni aspetti della vendita e delle garanzie concernenti i beni di consumo, al fine di garantire un livello minimo uniforme di tutela dei consumatori nel quadro del mercato interno. 2 . Ai fini della presente direttiva si intende per: a) consumatore: qualsiasi persona fisica che, nei contratti soggetti alla presente direttiva, agisce per fini che non rientrano nell'ambito della sua attività commerciale o professionale; b) beni di consumo: qualsiasi bene mobile materiale tranne: $-i$ beni oggetto di vendita forzata o comunque venduti secondo altre modalità dalle autorità giudiziarie; - l'acqua ed il gas, quando non confezionati per la vendita in un volume delimitato o in quantità determinata; - l'energia elettrica; c) venditore: qualsiasi persona fisica o giuridica che in base a un contratto vende beni di consumo nell'ambito della propria attività commerciale o professionale; d) produttore: il fabbricante di un bene di consumo, l'importatore del bene di consumo nel territorio della Comunità europea o qualsiasi altra persona che si presenta come produttore apponendo sul bene di consumo il suo nome, marchio o altro segno distintivo; e) garanzia: qualsiasi impegno di un venditore o di un produttore, assunto nei confronti del consumatore senza costi supplementari, di rimborsare il prezzo pagato, sostituire, riparare, o intervenire altrimenti sul bene di consumo, qualora esso non corrisponda alle condizioni enunciate nella dichiarazione di garanzia o nella relativa pubblicità; f) riparazione: nel caso di difetto di conformità, il ripristino del bene di consumo per renderlo conforme al contratto di vendita. 3. Gli Stati membri possono stabilire che nei «beni di consumo» non sono inclusi beni usati, venduti in un'asta alla quale il consumatore abbia la possibilità di assistere personalmente. 4 . Ai fini della presente direttiva sono considerati contratti di vendita anche i contratti di fornitura di beni di consumo da fabbricare o produrre.

${ }_{17}$ Al riguardo vale la pena ricordare non è stata mai accettata la mozione del Comitato economico e sociale a proposito della 'Proposta di Direttiva del Parlamento europeo e del Consiglio sulla vendita e le garanzie dei beni di consumo', in G.U.C.E., n. C 307, del 16 ottobre 1996, 8, nel parere del 27 novembre 1996, in G.U.C.E., n. C 66, del 3 marzo 1997, \$. 3.4, di includere nella definizione di consumatore, «non solo le persone fisiche ma anche quelle giuridiche a condizione che queste ultime non operino nel quadro della loro attività economica e professionale per evitare una disparità di trattamento nella protezione garantita dalla Direttiva». A tal proposito cfr. M.P. Mantovani, $L a$ vendita dei beni di consumo, cit., 57. 
Vengono dunque delimitati i confini dell'ambito soggettivo della nozione di consumatore che non si presenta consona alla logica protettiva che costituisce la ratio della Direttiva 1999/44/CE ${ }^{18}$.

Può dunque essere considerato consumatore la persona fisica che agisce, nei contratti di vendita e negli altri contratti mobiliari, con finalità che siano estranee alla propria attività professionale o commerciale. D'altro canto, non può essere reputato consumatore colui che stipuli un contratto di vendita con lo scopo di assicurarsi la disponibilità di un bene da impiegare nello svolgimento della propria attività professionale per necessità personali, o chi acquisisca un bene indispensabile per l'avviamento della propria attività imprenditoriale. La Direttiva 1999/44/CE, inoltre, non riguarda le vendite di natura mista, professionale e personale, e nemmeno i negozi di natura preparatoria ${ }^{19}$.

La suddetta concezione di consumatore viene ribadita anche dalla giurisprudenza comunitaria, in cui emerge una nozione di consumatore come persona fisica che agisce per motivazioni di natura personale o familiare ${ }^{20}$.

Emerge chiaramente che il parametro identificativo della figura del consumatore coincide con le finalità oggettive dell'atto e dei beni negoziati, senza che rilevino le intenzioni soggettive del contraente e le motivazioni che lo hanno spinto a concludere il negozio.

Quanto poi alla nozione di venditore, nella Direttiva viene definito come 'qualsiasi persona fisica o giuridica che in base a un contratto vende beni di consumo nell'ambito della propria attività commerciale o professionale' (art. $1, \S .2$, let. c), da cui consegue che nell'ipotesi di un contratto concluso da un soggetto al di fuori della propria attività imprenditoriale o professionale il provvedimento comunitario non trova applicazione ${ }^{21}$. Va inoltre specificato, che la Direttiva non si applica ai contratti di trasferimento di beni di consumo

${ }_{18}$ In merito a ciò, si veda: Parlamento europeo, Documento di lavoro della Commissione per il mercato interno e la protezione dei consumatori sul diritto contrattuale europeo e la revisione dell'acquis: situazione attuale e nozione di consumatore, del 17 maggio 2006.

${ }_{19}$ Cfr. G. De Cristofaro, Difetto di conformità al contratto e diritti del consumatore. L'ordinamento italiano e la Direttiva 1999/44/CE sulla vendita e le garanzie dei beni di consumo, Padova, 2000, 37 s.

${ }^{20}$ Così come emerge in Cort. Giust. CE, 3 luglio 1997, C-269/95, Benincasa c. Dentalkit, nello specifico si veda il punto n. 16, in Giust. civ., I, 1999, 13, con nota di U. Corea, in cui si legge: «Al fine di stabilire lo status di consumatore di una persona, nozione che va interpretata restrittivamente, occorre riferirsi al ruolo di tale persona in un contratto determinato, rispetto alla natura ed alla finalità di quest'ultimo, e non invece alla situazione soggettiva di tale stessa persona. [...] un solo e medesimo soggetto può essere considerato un consumatore nell'ambito di determinate operazioni ed un operatore economico nell'ambito di altre».

${ }^{21}$ Rinvio a tale proposito alla Relazione alla proposta di Direttiva presentata dalla Commissione, in COM (1995) 520 def., in cui si evidenzia che «la presente proposta concerne soltanto le vendite effettuate da venditori professionali. Le vendite private sfuggono quindi completamente al suo campo d'applicazione e restano interamente sottoposte ai regimi nazionali applicabili». 
per scopi non professionali, anche se il bene sia acquistato per usi di tipo professionale ${ }^{22}$.

\section{La tutela del compratore/consumatore nella Direttiva 1999/44/CE: la garanzia per i vizi della cosa venduta}

La Direttiva 1999/44/CE ha tratteggiato anche il concetto di garanzia e le sanzioni a tutela del consumatore. Ai sensi dell'art.1 lett. e) la nozione di garanzia viene così descritta: "qualsiasi impegno di un venditore o di un produttore, assunto nei confronti del consumatore senza costi supplementari, di rimborsare il prezzo pagato, sostituire, riparare, o intervenire altrimenti sul bene di consumo, qualora esso non corrisponda alle condizioni enunciate nella dichiarazione di garanzia o nella relativa pubblicità".

Nell'art. 2, §.1, della Direttiva si legge inoltre che 'il venditore deve consegnare al consumatore beni conformi al contratto di vendita'. Tale previsione appare in prima battuta piuttosto scontata e non particolarmente significativa, dato che è oramai pacifico che venga riconosciuto al compratore/consumatore il diritto di ottenere beni coincidenti con quanto pattuito nel contratto di compravendita (o di appalto). Come giustamente specificato nel Considerando n. 7 della Direttiva 1999/44/CE: «il principio di conformità al contratto può essere considerato come una base comune alle varie tradizioni giuridiche nazionali». Lart. 2, \$. 2, prescrive le regole di integrazione del contratto, i cui contenuti traggono spunto all'art. 35 della Convenzione di Vienna e dalle normative nazionali europee più recenti $\mathrm{i}^{23}$.

Come stabilito dall'art. $2, \$$. 2, let. d), le dichiarazioni pubbliche del venditore, del produttore o del suo ausiliario contenute specificamente nella pubblicità $\mathrm{o}$ sull'etichettatura sono rilevanti ai fini dell'indicazione della presenza o l'assenza di determinate qualità del bene. Pertanto, le dichiarazioni pubbliche del venditore hanno il valore di una promessa contrattuale, anche nel caso in cui siano state manifestate da un terzo ${ }^{24}$.

Tale disposto normativo, che sanziona il comportamento del venditore, creando al contempo una tutela forte del consumatore, è plasmato sulla base dei Principles of European Contract Law ${ }^{25}$, dove è prevista una norma (l'art. 6:101, \$. 2

\footnotetext{
${ }^{22}$ Al riguardo si veda G. De Cristofaro, Difetto di conformità, cit., 40.

${ }^{23}$ Cfr. la Relazione alla proposta di Direttiva presentata dalla Commissione, in COM (1995) 520 def., del 18 giugno 1996, 11.

${ }^{24}$ Al riguardo, rinvio a D. Corapi, La direttiva 99/44/CE e la Convenzione di Vienna sulla vendita internazionale dei beni immobili, in Europa e diritto privato, V, 2002, 659.

${ }^{25}$ Si fa riferimento in questo contesto ai lavori portati avanti nel tempo (dal 1982 al 2003) dalla Commission on European Contract Law (designata comunemente anche come Commissione Lando).
} 
PECL $)^{26}$ in cui si stabilisce, fra le dichiarazioni di una parte che danno origine ad una obbligazione contrattuale, che sono ricomprese 'le informazioni sulla qualità o l'utilizzo di servizi, merci o altri beni' che un fornitore professionista rende note con finalità pubblicitarie o di commercializzazione delle merci in un momento antecedente alla conclusione del contratto.

Inoltre, nella Direttiva sono previste delle disposizioni innovative in merito alle conseguenze che deriverebbero dalla mancata corrispondenza del bene a ciò che è stato stabilito dalle parti nel contratto, delineando così i presupposti della responsabilità del professionista per il 'difetto di conformità del bene di consumo' e la corrispondente regolamentazione delle pretese del compratore/ consumatore nel caso in cui si manifesti tale difformità.

La Direttiva prevede anche un c.d. parametro relazionale per l'identificazione delle diverse tipologie di difetti, che si sostanzia nella comparazione tra l'essenza del bene nella sua fisicità e l'essenza del bene c.d. 'letterale' come deriva dalla descrizione fornita dalle parti al momento della conclusione del contratto. Il legislatore europeo ha rimpiazzato il metodo tradizionale di accertamento al modello 'ideale' di res, con un criterio che rimette alle parti la delimitazione delle caratteristiche del bene, consentendo così al compratore/consumatore una dimostrazione più agevole del fatto che l'oggetto di vendita non coincide con le caratteristiche delineate dalle parti ed inserite nel contratto.

Pertanto, ogni qualvolta il bene oggetto di compravendita, anche già consegnato dal venditore al compratore/consumatore, non presenti anche una sola delle caratteristiche stabilite dalle parti nell'accordo contrattuale, esso sarà qualificato in difetto di conformità, senza che rilevi l'importanza dell'infrazione delle regole contrattuali.

Il venditore sarà ritenuto responsabile per inadempimento se il difetto di conformità sia presente alla della consegna dell'oggetto di vendita e si riveli al compratore/consumatore nell'arco temporale dei due anni successivi dall'avvenuta compravendita.

Si tratta di una commissione non governativa, costituita prevalentemente da giuristi provenienti dai diversi Paesi dell'Unione europea, presieduta dal Prof. Ole Lando dell'Università di Copenaghen, dal 1982 lavora alla formulazione di Principi del diritto europeo dei contratti, Principles of European Contract law (PECL). Per le versioni italiane dei PECL vedi: C. Castronovo, in Europa e dir. priv., 2000, 249 ss.; G. Alpa, in Riv. crit. dir. priv., 2000, 498 ss. Rinvio anche a G. Luchetti, A. Petrucci, Fondamenti di diritto contrattuale europeo: dalle radici romane al progetto dei Principles of European Contract Law della Commissione Lando, Bologna, 2006.

${ }^{26}$ Si veda l'art. 6:101, \$. 2 PECL: (Contents and effects) If one of the parties is a professional supplier who gives information about the quality or use of services or goods or other property when marketing or advertising them or otherwise before the contract for them is concluded, the statement is to be treated as giving rise to a contractual obligation unless it is shown that the other party knew or could not have been unaware that the statement was incorrect. 
La Direttiva europea sulla vendita di beni di consumo, oltre all'azione redibitoria e all'azione estimatoria, dall'art. 3, \$. 2, ha previsto due nuove modalità di tutela del compratore che consentono un più rapido ed efficiente rapporto tra le parti contrattuali, in considerazione del particolare ruolo imprenditoriale del venditore. Nell'ipotesi in cui il bene consegnato al compratore/consumatore mostri anche un solo difetto di conformità ${ }^{27}$ rispetto a quanto statuito nel contratto, possono essere fatti valere i diritti riconosciuti al compratore/ consumatore, ovvero il diritto al ripristino, senza spese, della conformità del bene mediante riparazione o sostituzione, il diritto ad una riduzione adeguata del prezzo, nonché alla risoluzione del contratto relativo a tale bene.

Tale tutela viene concordata solo a colui che ha concluso il contratto di vendita ed unicamente verso il professionista con il quale il compratore/consumatore ha stipulato il contratto. Al compratore/consumatore, inoltre, non viene accordata una piena libertà in merito al rimedio di tutela da intentare in presenza di difetto di conformità del bene: il ricorso a tre dei quattro rimedi previsti dalla Direttiva è condizionato dalla sussistenza di criteri oggettivi.

Non trovarono, infatti, accoglimento gli emendamenti presentati dal Parlamento europeo volti ad estendere la legittimazione passiva anche al produttore dei beni di consumo ${ }^{28}$, attribuendo al consumatore un'azione diretta verso quest'ultimo.

La Direttiva stabilisce, inoltre, una gerarchia tra i differenti strumenti di tutela che prevede la riparazione e la sostituzione del bene al primo posto, la riduzione

27 Sulla valenza innovativa del principio di conformità previsto nella Direttiva 1999/44/CE, e il recepimento di tale principio nella disciplina della vendita ad opera del legislatore italiano rinvio a $\mathrm{G}$. Amadio, La 'conformità al contratto' tra garanzia e responsabilità, in Contr. impr. Europa, I, 2001, 5 ss.; Id., Difetto di conformità e tutele sinallagmatiche, in Riv. dir. civ., I, 2001, 880 ss. 873 s.; E. Corso, La tutela del consumatore dopo il decreto legislativo di attuazione della direttiva 99/44/CE, in Contr. impr. Europa, III, 2002, 1350. Più recente, L. Buffoni, La garanzia di conformità nel consumo, in La responsabilità per prodotto difettoso e la garanzia di conformità nel codice del consumo, II, a cura di P. Bortonem, L. Buffoni, Torino, 2007, 140.

${ }^{28}$ Si vedano al riguardo gli emendamenti nn. 3, 4, 5, 10 e 25 approvati dal Parlamento nella Risoluzione legislativa recante il Parere del Parlamento sulla Proposta di Direttiva del 1996. Va ricordato che anche il Comitato economico e sociale al \$. 2.5 della Proposta di Direttiva del 1996 aveva sollecitato il riconoscimento al consumatore del diritto di rivolgersi direttamente al produttore. Successivamente, in occasione della redazione della Proposta modificata del 1998, COM (1998) 217 def., p. 4, del 31 marzo 1998, in G.U.C.E., n. C 148, del 14 maggio 1998, 12, gli emendamenti del parlamento citati supra sono stati respinti in blocco dalla Commissione dichiarando che è preferibile lasciare la possibilità agli Stati membri di prevedere la responsabilità diretta del produttore e che «i diritti del consumatore, ed in generale il sistema attuale della Direttiva, non sono idonei all'introduzione di tale approccio». Tale scelta della Commissione è stata poi ribadita dal Consiglio, che ha ritenuto «necessario un ulteriore studio prima di prendere in considerazione l'integrazione di questo aspetto nella Direttiva». A tale proposito, vale la pena di mettere a confronto la Motivazione della posizione comune del Consiglio del 24 settembre 1998, in G.U.C.E., n. C 333 del 30 ottobre 1998, 46. 
del prezzo e la risoluzione del contratto al secondo posto, a cui seguono i rimedi sussidiari, esperibili in subordine all'esperimento dei primi ${ }^{29}$.

La proposizione di questa struttura gerarchica, che prevede la supremazia della coppia di rimedi sostituzione/riparazione, rispetto alla coppia di rimedi riduzione/risoluzione, attesta un progressivo distacco dalla tradizione romanistica, in cui, come noto e come è stato già ricordato in precedenza, era prevista la concessione delle azioni edilizie a tutela del compratore sulla base della coppia di rimedi risoluzione del contratto/riduzione del prezzo in qualità di sanzioni derivanti dall'actio redhibitoria e dall'actio aestimatoria.

E inoltre necessario rilevare che in merito alla disciplina ed alla nozione di risoluzione del contratto e riduzione del prezzo, i dati contenuti nella Direttiva mostrano carenze, e pertanto si deve fare necessariamente riferimento alla regolamentazione giuridica prevista in materia sia dalla Convenzione di Vienna e sia dalle legislazioni nazionali ${ }^{30}$.

Come nel diritto romano, il diritto alla riduzione del prezzo coincide con il diritto del compratore/consumatore di conseguire la diminuzione della somma di denaro da corrispondere a titolo di prezzo a seguito della consegna del bene di consumo oggetto di contratto. Inoltre, sempre in relazione alla riduzione del prezzo, la Direttiva statuisce che essa sia 'adeguata', e si presume, che questo criterio di adeguatezza debba corrispondere non solo alle esigenze del compratore/consumatore ma anche a quelle del venditore/imprenditore.

Il diritto alla risoluzione del contratto, invece, si sostanzia nello scioglimento del rapporto contrattuale instaurato a seguito della conclusione del contratto di compravendita, da cui consegue per le parti l'estinzione dei diritti e doveri derivanti dall'accordo contrattuale, e l'obbligo di restituzione di ciò che si è ricevuto a seguito dell'esecuzione del contratto. Appare evidente lo stretto collegamento con la disciplina romanistica derivante dall'esperimento dell'azione edilizia redibitoria.

\section{Considerazioni conclusive}

Dal breve excursus tracciato in questo contributo, appare evidente come la Direttiva 1999/44/CE, su taluni aspetti della vendita di beni di consumo, rappresenti l'espressione più significativa del ruolo che il legislatore europeo detiene nel campo della protezione del compratore/consumatore in materia contrattuale.

${ }^{29}$ Cfr. A.M. Mancaleoni, I contratti con i consumatori tra diritto comunitario e diritto comune europeo, Napoli, 2005, 119.

${ }^{30}$ Cfr. G. De Cristofaro, Difetto di conformità, cit., 198. 
Come è stato evidenziato, essa non concerne soltanto alla realizzazione di un'armonizzazione delle legislazioni nazionali, ma inserisce nel quadro della disciplina della materia una nuova forma di tutela specifica in riferimento alla figura del compratore/consumatore, facendo conseguentemente emergere rapidamente una rilevanza nell'economia di mercato dei contratti posti in essere da quest'ultimo. Tali considerazioni possono essere riferite anche a tutte le altre Direttive europee che si sono occupate della protezione del consumatore, le quali, insieme alla Direttiva 1999/44/CE, hanno ulteriormente contribuito a delineare quella che viene dai più definita come "disciplina consumeristica".

Il campo in cui questi provvedimenti europei si posizionano può ritenersi, sicuramente, innovativo da vari angoli di visuale, soprattutto nella valutazione dell'efficacia sui differenti livelli di intervento normativo: un livello orizzontale di intervento, coincidente con le differenti normative nazionali, e un livello verticale, inserito dall'alto conseguentemente all'adeguamento normativo imposto dalle direttive europee.

L'efficacia di intervento verticale della disciplina per i vizi della cosa venduta si riscontra non solo nella portata dell'Editto degli edili curuli nell'antica Roma, ma anche nella Direttiva 1999/44/CE oggetto di studio, dove l'intervento normativo è giustificato dall'esigenza di creare uno spazio di tutele maggiori destinate a determinati soggetti, nonché delimitate per contenuto e ambito.

Ancora una volta, come dimostrato anche in questo contributo, la modalità di intervento elaborata dagli edili curuli in età repubblicana, i quali intervennero al fine di riportare l'aequitas tra le parti contrattuali del contratto di compravendita, in merito alla garanzia dei vizi occulti della cosa venduta, appare di sorprendente modernità.

\section{Summary}

Nel presente contributo si sono voluti comparare taluni aspetti della disciplina della tutela del contraente debole nel contratto di compravendita, figura che attualmente coincide con quella del consumatore, concentrando l'attenzione su alcuni istituti presenti nel diritto romano, in cui emergono alcune situazioni di disparità contrattuale.

Nella disciplina dell'Editto degli edili curuli, i magistrati repubblicani stabilirono una normativa speciale per la dichiarazione dei vizi occulti nelle vendite di schiavi ed animali, presupponendo una responsabilità oggettiva del venditore che non si fosse attenuto alle disposizioni edilizie. Un intervento importante che andò a definire una settorializzazione della materia, inquadrata all'interno della vendita in generale. Da ciò derivava una sovrapposizione in livelli della disciplina giuridica negoziale: sul piano orizzontale si collocava la regolamentazione della vendita prevista dal ius civile, mentre sul piano verticale si innestava quella speciale, di ius honorarium, prevista dall'Editto degli edili curuli. Tale modalità si riscontra anche in ambito europeo con la Direttiva 1999/44/CE, a proposito della vendita di beni di consumo, che rappresenta la manifestazione più eclatante del ruolo che il legislatore europeo ha nell'ambito della protezione del consumatore in materia contrattuale. Come nel mondo antico, nella Direttiva 1999/44/CE l'intervento normativo 
è giustificato dall'esigenza di creare uno spazio di tutele maggiori destinate a determinati soggetti, nonché delimitate per contenuto e ambito

Parole chiave: Editto degli edili curuli, tutela del compratore e del consumatore; Direttiva 1999/44/CE

\section{The origins of consumer's legal protection: roman foundations of european discipline}

\section{Summary}

This set to compare certain aspects of weaker contractor's protections in the contract of sale, which are currently coinciding with those of the consumer, focusing on some instances resent in the Roman law, which deal with contractual disparity.

In the Edict of Curuli, the Republican magistrates established special rules for declaring consealed vices in slave and animal sales, assuming an objective liability of the seller who did not abide by the regulations. An important intervention that defined sectoralization of that matter, framed within the sale in general. This resulted in an overlap of planes in legal negotiation framework framework: on he horizontal level, the regulation of the sale was on by the government (ius civile), while on the vertical plane, the local magistrate (ius honorarium, on the basis of the edict was incorporated. This is also the case with the European Directive 1999/44 / EC on the sale of consumer goods, which is the most striking manifestation of the European legislator's role in consumer protection in contractual matters. As in the ancient world, in Directive 1999/44 / EC regulatory action is justified by the need to create a larger protection area for certain subjects, as well as delimited by content and scope.

Keywords: Edict of the Aediles Curules; Buyer and consumer protection; Directive 1999/44 / EC. 